\title{
Feasibility and validity of transcranial duplex sonography in patients with acute stroke
}

\author{
T Gerriets, M Goertler, E Stolz, T Postert, U Sliwka, F Schlachetzki, G Seidel, S Weber, \\ $M$ Kaps, for the Duplexsonography In Acute Stroke (DIAS) study group
}

J Neurol Neurosurg Psychiatry 2002;73:17-20

See end of article for authors' affiliations

......................

Correspondence to: Professor M Kaps, Am Steg 20, D-35385 Giessen, Germany;

manfred.kaps@

neuro.med.uni-giessen.de

Received

22 December 2000

In final revised form

28 November 2001

Accepted

8 February 2002 the feasibility of transcranial colour coded Objectives: To evaluate in a prospective multicentre setting the feasibility of transcranial colour coded
duplex sonography (TCCS) for examination of the middle cerebral artery (MCA) in patients with acute duplex sonography (TCCS) for examination of the middle cerebral artery (MCA) in patients with acute
hemispheric stroke, and to assess the validity of sonographic findings in a subgroup of patients who also had a correlative angiographic examination.

Methods: TCCS was performed in 58 consecutive patients within six hours of the onset of a moderate to severe hemispheric stroke. Ultrasound contrast agent (Levovist) was applied if necessary. Thirty two patients also had computed tomography angiography $(n=13)$, magnetic resonance angiography $(n=18)$, or digital subtraction angiography $(n=1)$. In 14 of these patients, both the sonographic and corresponding angiographic examination were performed within six hours of stroke onset (mean time difference between TCCS and angiography 0.8 hours). Eighteen patients, in whom angiography was carried out more than 24 hours after stroke onset, had a follow up TCCS for method comparison (mean time difference 6.1 hours).

Results: Initial unenhanced TCCS performed 3.4 (SD 1.2) hours after the onset of symptoms depicted the symptomatic MCA mainstem in 32 patients (55\%) (13 occlusions, one stenosis, 18 patent arteries). After signal enhancement, MCA status could be determined in 54 patients $(93 \%)(p<0.05)$, showing an occlusion in 25, a stenosis in two, and a patent artery in 27 patients. In 31 of the 32 patients who had correlative angiography, TCCS and angiography produced the same diagnosis of the symptomatic MCA (10 occlusions, three stenoses, 18 patent arteries); TCCS was inconclusive in the remaining one.

Conclusion: TCCS is a feasible, fast, and valid non-invasive bedside method for evaluating the MCA in an acute stroke setting, particularly when contrast enhancement is applied. It may be a valuable and cost effective alternative to computed tomography and magnetic resonance angiography in future stroke trials.
D uring the last decade, a considerable number of new treatments have emerged for acute stroke. Considering the current debate about whether certain subgroups of patients may benefit more than others from thrombolytic or neuroprotective treatments, fast, non-invasive, and cost effective diagnostic methods are of increasing interest. ${ }^{1}$ However, these methods need to fit into emergency acute stroke management.

Several studies have evaluated transcranial colour coded duplex sonography (TCCS) for the assessment of intracranial haemodynamics in stroke patients..$^{2-8}$ However, their predictive power with respect to the acute phase of stroke-that is, within six hours of symptom onset-has been limited by the small number of patients investigated, the low frequency of pathological findings, or the lack of a reference method (table 1). Furthermore, none of the previously published studies was designed as a multicentre trial, which may lead to bias because of differences in individual examination techniques.

We therefore launched the Duplexsonography In Acute Stroke (DIAS) study to clarify the potentials and limitations of TCCS in this respect. The investigation presented here was carried out to evaluate the feasibility and validity of TCCS in detecting intracranial stenosis and occlusion in patients with an acute moderate to severe hemispheric stroke within a six hour time window in a prospective multicentre setting.

\section{MATERIALS AND METHODS}

The DIAS study was carried out prospectively in eight centres in Germany and Switzerland over nine months. It was approved by a local ethics committee. Eligible patients were men and women aged over 18 years with clinical signs of a moderate to severe hemispheric stroke (Scandinavian stroke scale score $<50$ ), who were admitted within six hours of onset of symptoms.

Extracranial and transcranial colour coded duplex sonography were performed immediately on admission in each patient. Patients in whom correlative angiography could not be carried out within six hours of stroke onset had follow up sonography for method comparison ensuring a less than 24 hour time span between the two techniques. Ultrasound devices used by the eight centres were an HP SONOS 2000, 2500 or 5500, an Acuson 128xP/10, a Toshiba SSH-140 HG or SSH-380, or a Siemens Elegra. All ultrasound examinations were performed by doctors with at least one year of experience in the field of colour coded duplex sonography of the brain-supplying arteries. The sonographers were not aware of the patient's stroke scale score, computed tomography data, and angiography.

The extracranial internal carotid artery (ICA) and common carotid artery were classified as "normal", "stenotic $<70 \%$ ", "stenotic $>70 \%$ " or "occluded" according to previously

Abbreviations: TCCS, transcranial colour coded duplex sonography; ICA, internal carotid artery; MCA, middle cerebral artery; ACA, anterior cerebral artery; PCA, posterior cerebral artery; CTA, computed tomography angiography; MRA, magnetic resonance angiography; DSA, digital subtraction angiography 
Table 1 Studies on transcranial colour coded duplex sonography for the assessment of intracranial haemodynamics in patients with ischaemic anterior hemispheric stroke

\begin{tabular}{|c|c|c|c|c|c|}
\hline Reference & $\begin{array}{l}\text { Number of } \\
\text { patients }\end{array}$ & $\begin{array}{l}\text { Time since stroke } \\
\text { onset }\end{array}$ & $\begin{array}{l}\text { MCA occlusion (M1 } \\
\text { segment) }\end{array}$ & Reference method & $\begin{array}{l}\text { Patients with } \\
\text { reference method }\end{array}$ \\
\hline Seidel et al. $(1995)^{2}$ & 48 & $\leqslant 48$ hours & 15 & n.d. & 0 \\
\hline Baumgartner et al. (1997) 3 & 33 & n.a. & 0 & DSA & 6 \\
\hline Kenton et al. $(1997)^{5}$ & 39 & $\leqslant 24$ hours & 10 & MRA & 30 \\
\hline Goertler et al. $(1998)^{6}$ & 23 & $\leqslant 5$ hours & 4 & DSA & 1 \\
\hline Gahn et al. (1999)7 & 34 & $\leqslant 21$ days* & 3 & CTA & 34 \\
\hline Gerriets et al. (1999) ${ }^{4}$ & 47 & $\leqslant 12$ hours $\dagger$ & 18 & DSA/MRA & 11 \\
\hline Postert et al. (1999) ${ }^{8}$ & 90 & $\leqslant 12$ hours & 20 & CTA & 39 \\
\hline Present study & 58 & $\leqslant 6$ hours & 25 & MRA/CTA/DSA & 32 \\
\hline
\end{tabular}

*Estimated from mean (SD) 8.1 (6.6) days; †most patients.

MCA, Middle cerebral artery; n.d., not done; n.a., not available; DSA, digital subtraction angiography; MRA, magnetic resonance angiography; CTA computed tomography angiography.

published criteria.9 A "preocclusive signal" was diagnosed if extracranial duplex sonography of the ICA showed a typical high resistance signal with absence of diastolic flow. ${ }^{4}$

TCCS was performed through the left and right temporal acoustic bone window with 2.0-2.5 MHz sector transducers as described previously (fig 1)..$^{2-4}$ Middle cerebral artery (MCA) mainstem occlusion was diagnosed when no Doppler signal could be obtained in the lateral fissure while the anterior and posterior cerebral arteries ( $\mathrm{Pl}$ or $\mathrm{P} 2$ segment) were sufficiently assessable (fig 1). ${ }^{34}$ An (additional) occlusion of the intracranial ICA (carotid $\mathrm{T}$ occlusion) was considered when the intracranial portion of the ICA was assessable by TCCS but no colour signal or Doppler spectrum could be obtained, or when extracranial duplex sonography of the ICA showed a preocclusive signal. A stenosis of the MCA was ascertained when Doppler spectral analysis showed circumscribed acceleration of mean systolic blood flow velocity $(>120 \mathrm{~cm} / \mathrm{s})$, a side to side difference in blood flow velocity of more than $21 \%$, and spectral signs of disturbed flow. Angle correction was applied when the correction angle did not exceed $30^{\circ}$ and an appropriate straight vessel segment could be identified. ${ }^{10-12}$ Side to side comparison thereby compensates the velocity increase observed after signal enhancement, ${ }^{313}$ as blood flow velocities were obtained from contrast enhanced measurements if available.

Investigation of an artery - that is, the MCA (ipsilateral and contralateral Ml segment), anterior cerebral artery (ACA; ipsilateral Al), posterior cerebral artery (PCA; ipsilateral Pl or $\mathrm{P} 2$ ), and the ipsilateral distal ICA-was considered satisfactory if the corresponding segment was visible to its full extent and an unambiguous diagnosis could be obtained. Otherwise contrast enhancement (Levovist; Schering, Berlin, Germany) was used: one vial (4 g) Levovist suspension was administered at a concentration of $300-400 \mathrm{mg} / \mathrm{ml}$ as a single or multiple bolus injection or infusion. The duration of the complete extracranial and intracranial duplex examination was determined offline from videotapes.

The choice of the correlative method-computed tomography angiography (CTA), magnetic resonance angiography (MRA), or digital subtraction angiography (DSA) — was left up to the treating centre. DSA and CTA, the latter by the use of the spiral scanning technique, were predominantly performed at three centres. Five centres predominantly used MRA. Vessel diagnosis with CTA and MRA in all centres was obtained by the evaluation of source slices combined with three dimensional maximum intensity projection reconstruction. The angiographic investigator who assessed the examination immediately after it had been performed was blinded to the results of the sonography and vice versa.

The number of sufficiently assessable basal cerebral arteries before and after the additional administration of contrast agent was compared using Wilcoxon's matched pairs signed rank test. Differences in the time to complete the ultrasound examination among the participating centres were compared using the Kruskal-Wallis test. For method comparison, Pearson's $\chi^{2}$ test was used. A probability level of $\mathrm{p}<0.05$ was considered significant.

\section{RESULTS}

Fifty eight consecutive patients ( 36 men, 22 women) admitted within six hours of the onset of a moderate to severe hemispheric stroke were included in this study. Median age was 64 years (range 38-89), and the median Scandinavian stroke scale score upon admission was 26 (range 4-48).

The initial TCCS examination was performed 3.4 (SD 1.2) hours after the onset of symptoms. For satisfactory investigation of the entire ipsilateral circle of Willis (ipsilateral MCA,
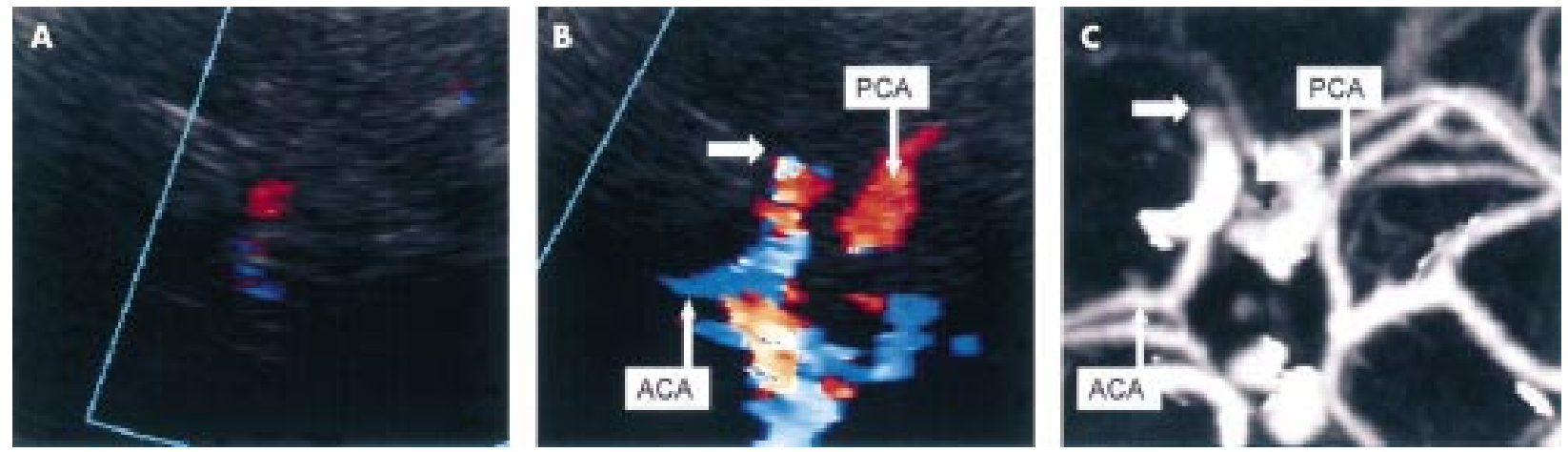

Figure 1 Transcranial colour coded duplex sonography (TCCS) and computed tomography angiography of a patient, with an acute right hemiparesis, with insufficient transtemporal ultrasound penetration. (A) Unenhanced TCCS was inconclusive. (B) After contrast enhancement (4 $g$ Levovist; bolus application), the anterior (ACA) and posterior cerebral arteries (PCA) and the contralateral middle cerebral artery (MCA) became visible. No Doppler signal could be obtained in the left lateral fissure, indicating a middle cerebral artery (MCA) occlusion (arrow). (C) The computed tomography angiogram confirmed the MCA occlusion (arrow). 


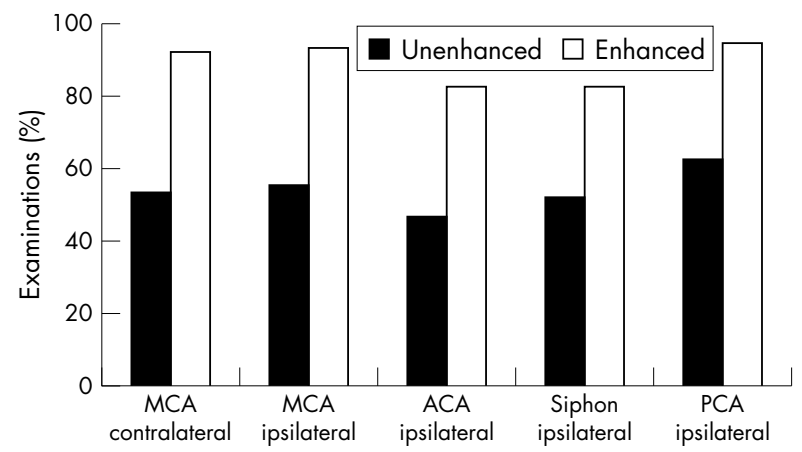

Figure 2 Percentage of sufficiently assessable arterial segments of the circle of Willis before and after additional administration of Levovist in 58 patients investigated within six hours of stroke onset. MCA, ACA, and PCA indicate middle, anterior, and posterior cerebral artery.

ICA, ACA, and PCA) and the contralateral MCA, contrast enhancement was considered necessary in 51 of the 58 patients by the sonographic examiner. No adverse effects occurred after Levovist application in this study. Diagnosis of the symptomatic MCA could be obtained in 32 of 58 patients (55\%) before, and in 54 of 58 patients (93\%) after, the additional use of contrast enhancement. Examinations before the administration of contrast enhancement showed an MCA occlusion in 13, an MCA stenosis in one, and a patent artery in 18 patients. After application of Levovist, 25 occlusions, two stenoses, and 27 patent arteries were diagnosed. Of the total of 290 basal cerebral arteries examined in the 58 patients, diagnostic investigation was possible in $160(55 \%)$ before and 260 $(90 \%)$ after application of contrast enhancement. Percentages were significantly different before and after contrast enhancement for the total number as well as for each vessel investigated (fig 2).

Mean duration of the complete initial extracranial and intracranial duplex examination (common, internal, and external carotid artery, vertebral artery, MCA, ACA, PCA, and intracranial portion of the ICA on both sides) was 21.3 minutes (SD 7.4; range 9-43; $\mathrm{n}=51$ ) in patients who received both unenhanced and contrast enhanced examinations, and 13.6 minutes ( SD 4.4; range 10-22; $\mathrm{n}=7$ ) in those who had an unenhanced investigation only. No significant differences could be detected among the eight participating centres with respect to the mean duration of the complete ultrasound examination $(\mathrm{p}=0.53)$.

Thirty two of the 58 patients received correlative angiography of the intracranial arteries (CTA in 13 patients, MRA in 18, DSA in 1). Reasons for not having a reference examination were critical illness preventing transportation (eight patients), restlessness resulting in extensive moving artefacts (six), MRA obstacles in five patients (implanted pace maker, massive adiposity, ankylosing spondylitis, technical scanner problems), early hemicraniectomy (one patient), and rapid clinical improvement (four patients). Two additional patients with angiographies were also excluded from the comparative investigation because the time between ultrasound examination and angiography exceeded 24 hours. In the 14 patients who had angiography within six hours of stroke onset, the mean time difference from TCCS was less than one hour (range 0.6-3.5; mean 0.8). In the 18 patients who had a follow up TCCS for method comparison because angiography was carried out more than 24 hours after stroke onset, mean time difference was 6.1 hours.

TCCS and correlative angiography resulted in identical diagnoses of the symptomatic MCA in 31 of the 32 patients (10 occlusions, three stenoses, 18 patent arteries). TCCS was inconclusive in one patient, despite contrast enhancement; this was because of strong ultrasound attenuation by the tem- poral bone. In 10 of the 32 patients, reference examination also included the extracranial part of the carotid system (three by CTA, six by MRA, one by DSA). Angiography in all patients confirmed the sonographic finding (three ipsilateral ICA occlusions, seven patent carotid arteries).

\section{DISCUSSION}

Our findings on the feasibility of unenhanced TCCS in stroke patients are different from those of most other investigations. Previous studies reported that ultrasonic attenuation by the temporal skull limited TCCS investigation in $10-20 \%$, and that the use of contrast enhancement can reduce the percentage of diagnostic failure to less than $10 \% \cdot{ }^{347}$ In our study, diagnostic investigation of the symptomatic MCA as well as the basal cerebral arteries was possible in only 55\% with unenhanced TCCS. This difference may be explained less by the slightly different mean age and proportion of women than by the acute (six hours) stroke setting, in which MCA occlusion is the predominant intracranial vascular finding ${ }^{14}$ with decreasing incidence in wider time windows (already at 24-48 hours $)^{15}{ }^{16}$ because of early vessel recanalisation. ${ }^{17}$ Reliable diagnosis of MCA occlusion and its differentiation from technical problems in the case of an undetectable flow signal is a well known problem of transcranial sonography and requires successful insonation of the uninvolved basal cerebral arteries. $^{25618}$ This may also explain the frequent (88\%) use of contrast enhancement considered necessary by the examiners to ensure reliable diagnosis of the symptomatic MCA. This assumption is confirmed by a recent study in which unenhanced TCCS performed within five hours of stroke onset enabled diagnosis of symptomatic MCA in only 52\% of patients. ${ }^{6}$ However, in that study and our study, reliable diagnosis was obtained in $87 \%$ and $93 \%$ respectively of patients after contrast enhancement. From our data, we conclude that the additional benefit of contrast enhancement in TCCS compared with unenhanced examination is maximal in an acute stroke setting with a limited time period and enables diagnosis of the symptomatic MCA in about $90 \%$ of patients.

The use of additional contrast enhancement in TCCS increased the time to complete the intracranial and extracranial examination from 14 to 21 minutes, which corroborates the results of a previous study. ${ }^{6}$ As the effect of contrast enhancement in TCCS is maximal in the very acute phase of stroke and its use was considered necessary for reliable diagnosis in $88 \%$ of consecutive patients included in this multicentre study, we suggest its early application in such patients before completion or even the start of unenhanced examination. This seems to be justified as no side effects of the agent used (Levovist) have been reported in this situation, and application may not be necessary in only one out of 10 patients. Without unenhanced transcranial examination and with assessment of only clinically relevant arteries-that is, ipsilateral extracranial and intracranial ICA, MCA, ACA, PCA, and contralateral MCA) - the time for the whole extracranial and intracranial examination in our patients would have been reduced to less than 15 minutes, which is similar to, or even less than, the mean examination time of 20 minutes for an intracranial CTA or MRA (3-10 minutes acquisition time plus additional data processing and reading time). ${ }^{5}$ Considering the limited applicability of CTA and MRA because of various technical and logistic problems in 20 of 26 patients without correlative angiography-that is, about one third of all consecutive patients with moderate to severe acute strokeand the bedside applicability of TCCS, the latter may even be the more appropriate and time saving imaging technique.

The validity of TCCS for the diagnosis of stenosis or occlusion of the symptomatic MCA was evaluated from 32 of our 58 patients who received an additional correlative 
angiography. Fourteen of these patients had both examinations within six hours of stroke onset, whereas in the remaining 18 patients correlative examinations for method comparison were performed more than 24 hours after. However, findings did not differ between the two groups. TCCS diagnosis of the symptomatic MCA was confirmed by MRA (18 patients, five centres), CTA ( 13 patients, three centres), and DSA (one patient, one centre which also performed CTA) in 31 of 32 patients $(97 \%)$, regardless of the centre and the pathological finding (10 occlusions, three stenoses, 18 patent arteries). Studies that have compared TCCS with a reference method in an acute stroke setting are limited. Postert et al ${ }^{8}$ investigated 39 stroke patients within a 12 hour time window by TCCS and CTA. In 38 patients (97\%), CTA confirmed the TCCS diagnosis. MCA status was misdiagnosed in one patient with a partial temporal bone window.

With regard to this problem as discussed elsewhere, ${ }^{146}$ contrast enhanced TCCS is also a fast, valid, and reliable method when performed in a multicentre setting for evaluation of cerebrovascular status in acute stroke patients eligible for acute revascularisation or neuroprotective treatments. Its non-invasive character and bedside applicability may make it a valuable alternative to CTA and MRA in acute stroke patients and a useful tool for future multicentre stroke trials.

\section{ACKNOWLEDGEMENTS}

The DIAS study was supported by the Stiftung Deutsche SchlaganfallHilfe. Part of this study was supported by grants from the Verband Forschender Arzneimittelhersteller and the Deutsche Forschungsgemeinschaft (to T G)

The Duplexsonography In Acute Stroke (DIAS) study group consists of:

Dr T Gerriets, Dr E Stolz, Dr M Jauss, Dr J Allendörfer, Professor M Kaps, Department of Neurology, Justus Liebig University, Giessen, Germany

Dr M Goertler, Dr R Kross, Dr T Blaser, Dr S Krueger, Department of Neurology, University of Magdeburg, Germany

Dr T Postert, Dr S Meves, Dr J Federlein, Department of Neurology, St Josef Hospital, Ruhr University, Bochum, Germany

Dr U Sliwka, Department of Neurology; University of Jena, Germany

Professor U Bogdahn, Dr F Schlachetzki, Dr T Hoelscher, Department of Neurology, University of Regensburg, Germany

Dr G Seidel, Dr M Vidal-Langwasser, Dr C Algermissen, Department of Neurology, Medical University at Luebeck, Germany

Professor R Baumgartner, Department of Neurology, University of Zurich, Switzerland

Professor B Widder, Department of Neurology, Bezirkskrankenhaus Günzburg, Germany

Dr S Weber, Schering AG, Berlin, Germany

\section{Authors' affiliations}

T Gerriets, E Stolz, M Kaps Department of Neurology, Justus Liebig

University, Giessen, Germany

T Gerriets Department of Radiology, Kerckhoff Clinic Foundation, Bad Nauheim, Germany

M Goertler Department of Neurology, University of Magdeburg

Germany

T Postert, Department of Neurology, St Josef Hospital, Ruhr University, Bochum, Germany

U Sliwka, Department of Neurology; University of Hamburg, Germany

F Schlachetzki, Department of Neurology, University of Regensburg,

Germany

G Seidel, Department of Neurology; Medical University at Luebeck,

Germany

S Weber, Schering AG, Berlin, Germany

\section{REFERENCES}

1 Gerriets T, Postert T, Goertler M, et al. DIAS I: Duplex-sonographic assessment of the cerebrovascular status in acute stroke: a useful tool for future stroke trials. Stroke 2000;31:2342-5

2 Seidel G, Kaps M, Gerriets T. Potential and limitations of transcranial color-coded sonography in stroke patients. Stroke 1995;26:2061-6.

3 Baumgartner RW, Arnold M, Gonner F, et al. Contrast enhanced color-coded duplex sonography in ischemic cerebrovascular disease. Stroke 1997;28:2473-8.

4 Gerriets T, Seidel G, Fiss I, et al. Contrast-enhanced transcranial color-coded duplex sonography. Efficiency and validity. Neurology 1999:52: 1133-7.

5 Kenton AR, Martin PJ, Abbott RJ, et al. Comparison of transcranial color-coded sonography and magnetic resonance angiography in akute stroke. Stroke 1997;28:1601-6.

6 Goertler M, Kross R, Baeumer M, et al. Diagnostic impact and prognostic relevance of early contrast-enhanced transcranial color-coded duplex sonography in acute stroke. Stroke 1998:29:955-62.

7 Gahn G, Gerber J, Hallmeyer S, et al. Noninvasive assessment of the circle of Willis in cerebral ischemia: The potential of CT angiography and contrast enhanced transcranial color-coded duplexsonography. Cerebrovasc Dis 1999:9:290-4.

8 Postert T, Braun B, Meves S, et al. Contrast-enhanced transcrania color-coded sonography in acute hemispheric brain infarctions. Stroke 1999;30:1819-26.

9 De Bray JM, Glatt B. Quantification of atheromatous stenosis in the extracranial internal carotid artery. Cerebrovasc Dis 1995;5:414-26.

10 Lindegaard KF. Intracranial artery stenosis. In: Newell DW, Aaslid R, eds. Transcranial doppler. New York: Raven Press, 1992:161-6.

11 Ley-Pozo J, Ringelstein EG. Noninvasive detection of occlusive disease of the carotid siphon and middle cerebral artery. Ann Neurol 1990;28:640-7

12 Giller CA. Is angle correction correct? J Neuroimaging 1994:4:51-2.

13 Forsberg $\mathbf{F}$, Liu JB, Burns PN, et al. Artifacts in ultrasonic contrast agent studies. J Ultrasound Med 1994;13:357-65.

14 Fieschi C, Argentino C, Lenzi GL, et al. Clinical and instrumental evaluation of patients with ischemic stroke within the first six hours. $J$ Neurol Sci 1989:91:311-21.

15 Olsen TS, Skriver EB, Herning M. Cause of cerebral infarction in the carotid territory. Its relation to the size and the location of the infarct and to the underlying vascular lesion. Stroke 1985;16:459-66.

16 Fields WS, North RR, Hass WK, et al. Joint study of extracranial arterial occlusion as a cause of stroke. I. Organization of study an survey of patient population. JAMA 1968;203:955-60.

17 Ringelstein EB, Biniek R, Weiller C, et al. Type and extent of hemispheric brain infarction and clinical outcome in early and delayed middle cerebral artery recanalization. Neurology 1992;42:289-98.

18 Martin PJ, Pye IF, Abbott RJ, et al. Color-coded ultrasound diagnosis of vascular occlusion in acute ischemic stroke. J Neuroimaging 1995;5: 152-6. 